\title{
Oxidative Stress Due to Anesthesia and Surgical Trauma and Comparison of the Effects of Propofol and Thiopental in Dogs
}

\author{
Jae Yeon LEE ${ }^{1) *}$ \\ ${ }^{1)}$ Department of Veterinary Surgery, College of Veterinary Medicine, Research Institute of Veterinary Medicine, Chungnam \\ National University, Daejeon 305-764, Korea
}

(Received 9 May 2011/Accepted 12 December 2011/Published online in J-STAGE 26 December 2011)

ABSTRACT. The present study aimed to evaluate the effect of propofol and thiopental on the plasma oxidant-antioxidant profile in dogs undergoing surgery at doses used to induce anesthesia. The plasma total oxidant status (TOS) and oxidative stress index (OSI) levels increased significantly with time in both groups, whereas the plasma total antioxidant status (TAS) levels decreased with time in both groups. The OSI was significantly higher at the end of surgery than before induction of anesthesia in both groups. The TOS and OSI change ratio of propofol group were significantly lower than that of thiopental group. In conclusion, our findings show that propofol has antioxidant effects in dogs. Further studies need to be conducted to demonstrate the exact mechanism of oxidative stress due to anesthesia and surgery in dogs.

KEYWORDS: canine, oxidative stress, propofol, total antioxidant status, total oxidant status.

doi: 10.1292/jvms.11-0221; J. Vet. Med. Sci. 74(5): 663-665, 2012

Anesthesia and surgery of any kind, especially in case of surgery of abdominal viscera, are well-recognized as oxidative stress-inducing manipulations [7]. Oxidative stress can be described as an imbalance between the production of free radicals and antioxidant defense. Over the last several decades, it has become amply evident that oxidative stress, usually in the form of reactive oxygen species (ROS), is a critical pathogenic factor in the development of cardiovascular disease and other systemic diseases [2, 4].

Inhibition of ROS formation, scavenging ROS, or interfering with ROS pathogenic signaling pathways might be the potential ways to protect against oxidative stress. Propofol (2, 6-diisopropylphenol), a currently-available sedative and anesthetic agent, is chemically similar to the endogenous antioxidant $\alpha$-tocopheral (vitamin E) and, theoretically, should have similar properties [1]. Numerous studies have demonstrated antioxidant effects of propofol in vitro [9-11] and in vivo [6], but the effect of propofol on oxidant and antioxidant balance in dogs has not been fully evaluated.

This study was designed to evaluate the effect of propofol and thiopental, at doses used to induce anesthesia, on the plasma oxidant-antioxidant profile in dogs undergoing surgery.

Eighteen mongrel dogs (nine males and nine females), 4-6 years old, weighing 5-10 kg were used. All dogs were determined to be healthy on the basis of clinical examinations. The experimental and housing protocols were approved by the Chungnam National University Animal

*Correspondence to: Lee., J. Y., College of Veterinary Medicine, Chungnam National University, Daejeon 305-764, South Korea.

e-mail address: romanavet@hanmail.net

(C)2012 The Japanese Society of Veterinary Science
Care and Use Committee. The study was carried out 14 days after procuring the dogs. The dogs were kept in a quiet room to avoid any stress-inducing factors during this period. The dogs were fasted for 8-12 hr before the experiments and water was withheld $2 \mathrm{hr}$ before anesthesia in order to prevent any potential adverse effects, such as vomiting or regurgitating stomach contents during anesthesia or during the recovery period. The dogs had received given intravenous (IV) fluid therapy with Hartmann's Solution (Hartmann Solution ${ }^{\circledR}$, Daihan Pharm Co., Ltd., Korea) at the infusion rate of $60 \mathrm{ml} / \mathrm{kg} / 24 \mathrm{hr}$ for $12 \mathrm{hr}$ until starting the experiment.

The dogs were randomly divided into two groups according to the type of anesthestic agents used for the surgical procedure. In the thiopental group, anesthesia was induced with an IV bolus dose of thiopental $(12 \mathrm{mg} / \mathrm{kg}$ : Thionyl Injection ${ }^{\circledR}$, Daihan Pharm Co., Ltd.). In propofol group, an IV dose of $6 \mathrm{mg} / \mathrm{kg}$ of propofol (Aanepol Injection $^{\circledR}$, Hana Pharm Co., Ltd., Korea) was used. The dogs were premedicated with a subcutaneous injection of atropine sulfate $\left(0.04 \mathrm{mg} / \mathrm{kg}\right.$ : Atropine Sufate Injection ${ }^{\circledR}$, Daihan Pharm Co., Ltd.), an intramuscular injection of butorphanol $\left(0.2 \mathrm{mg} / \mathrm{kg}\right.$ : Butophan Injection ${ }^{\circledR}$, Myungmoon Pharm Co., Ltd., Korea), ranitidine (1 mg/kg, IV: Rnitidine HCL Injection ${ }^{\circledR}$, BCWP Pharm Co., Ltd.) and cefazolin $\left(20 \mathrm{mg} / \mathrm{kg}\right.$, IV: Falexin ${ }^{\circledR}$, Dong Wha Pharm Co., Ltd., Korea). Afterwards, thiopental sodium or propofol was administered for tracheal intubation. The anesthesia was maintained with $2 \%$ of end-tidal concentration of isoflurane (Forane Sol ${ }^{\circledR}$, Choongwae Pharm Co., Ltd., Korea) delivered with pure oxygen. Laparotomy and gastrotomy were performed to create surgical trauma in this study. Laparotomy and gastrotomy are common surgical procedures performed in veterinary practice and could be an important cause of oxidative stress by reduced abdominal visceral perfusion. A ventral midline celiotomy was per- 
Table 1. Oxidative stress parameters of the dogs

\begin{tabular}{ccccc}
\hline Parameters & Group & $\begin{array}{c}\text { Before induction of } \\
\text { anesthesia }\end{array}$ & End of the surgery & Change ratio (\%) \\
\hline TOS & Thiopental & $12.79(3.92)$ & $25.36(6.81)^{*}$ & $98.3(12.1)$ \\
$\left(\mu \mathrm{molH}_{2} \mathrm{O}_{2} / \mathrm{L}\right)$ & Propofol & $13.56(4.12)$ & $19.78(6.27)$ & $45.9(7.8)^{* *}$ \\
$\mathrm{TAS}^{*}$ & Thiopental & $1.38(0.34)$ & $0.88(0.28)$ & $-36.2(8.9)$ \\
$(\mathrm{mmol}$ Trolox equiv/L) & Propofol & $1.52(0.29)$ & $0.98(0.18)^{*}$ & $-35.5(9.7)$ \\
OSI & Thiopental & $10.44(4.25)$ & $37.22(7.01)^{*}$ & $256(31.1)$ \\
$($ arbitrary unit) & Propofol & $9.85(3.89)$ & $22.09(8.80)^{*}$ & $124(13.7)^{* *}$ \\
\hline
\end{tabular}

The values represent the median (inter-quartile range).

* Statistically difference compared to "before induction of anesthesia." ( $\mathrm{n}=9$ ).

${ }^{* *}$ Statistically difference compared to thiopental group $(\mathrm{n}=9)$.

formed. The body of stomach was grasped with atraumatic forceps or fingers and delivered into the ventral abdominal wall incision. And then, the incision was made in the center of the body (approximately 10-20 $\mathrm{mm}$ in length). The resultant incision was closed in an appositional, simple continuous pattern, and the second inverting layer was applied over this initial layer. The abdomen was closed in 3 layers. During the surgical operation, the dogs were given IV fluid (Hartmann's solution, $10 \mathrm{~m} / / \mathrm{kg} / \mathrm{h}$ ). The postoperative care was done. After surgery, each animal was monitored until it was ambulatory. Dogs were given butorphanol $0.2 \mathrm{mg} /$ $\mathrm{kg}$ intramuscularly at the end of the surgical procedure for postoperative analgesia. A second dose of butorphanol 0.1 $\mathrm{mg} / \mathrm{kg}$ was given intramuscularly $6 \mathrm{hr}$ after surgery. Water and moistened dog food were offered $12 \mathrm{hr}$ after surgery. Antibiotics (cefazolin, $20 \mathrm{mg} / \mathrm{kg}$, IV, bid) and ranitidine (1 $\mathrm{mg} / \mathrm{kg}, \mathrm{IV}$, bid) were administrated for 7 days, and antibiotics cream (Bactroban Ointment ${ }^{\circledR}$, Hanall Bio Pharm Co., Ltd., Seoul, Korea) was applied to the middle line area once daily for 13 days.

Blood samples $(3 \mathrm{ml})$ were collected via venipuncture from the jugular vein at each designated time. The samples were centrifuged at 3,000 rpm for $10 \mathrm{~min}$ to separate plasma and the blood samples were stored at $-80^{\circ} \mathrm{C}$ until analysis. Plasma total oxidant status (TOS) and total antioxidant status (TAS) levels for the evaluation of oxidative stress were determined using a commercially available kit (Mega Tip; REL Assay Diagnostics, Gaziantep, Turkey) developed by Erel [3]. The ratio of the TOS to the TAS gave oxidative stress index (OSI), an indicator of the degree of oxidative stress. The change ratio from each value was calculated as below;

TAS change ratio $=($ TAS End of the surgery - TAS Before induction of anesthesia) $\div$ TAS Before induction of anesthesia $\times 100$

TOS change ratio $=($ TOS End of the surgery - TOS Before induction of anesthesia $) \div$ TOS Before induction of anesthesia $\times 100$

OSI change ratio $=(\mathrm{OSI}$ End of the surgery $-\mathrm{OSI}$ Before induction of anesthesia $) \div$ OSI Before induction of anesthesia $\times 100$

Data were expressed as median (inter-quartile range), and Mann-Whitney U-test was used for detection of differences between blood sample. A p-value $<0.05$ was considered significant. All statistics were performed using a computer statistical package (Statistics Package for the Social Sciences, version 17.0; SPSS, Chicago, IL, U.S.A.).
Total anesthetic time was 110 (20) min (ranging from 80 $\min$ to $130 \mathrm{~min}$ ). Mean operation times of the thiopental and propofol groups were 33 (11) and 41 (15) min, respectively. The differences in the respective mean operation times of two groups were not stiatistically significant. All dogs were hemodynamically stable during experiments. There were no significant worsening in heart rate, mean arterial pressure, end-tidal carbon dioxide, peripheral oxygen saturation, and respiratory rates (data not shown).

The plasma TOS, TAS, and OSI are shown in Table 1. An increase in the plasma TOS levels in both groups after surgery was observed. The TOS level of the thiopental group was significantly higher at the end of surgery than before induction of anesthesia $(P=0.0002)$. Plasma TAS levels were decreased in both group after surgery. The TAS level of the propofol group was significantly lower at the end of surgery than before induction of anesthesia $(P=0.008)$. OSI levels increased significantly in the thiopental and propofol groups after surgery ( $P=0.0001$ and 0.008 , respectively). The TOS and OSI change ratio of propofol group were significantly lower than that of thiopental group $(P=0.004$ and 0.003 , respectively).

Hydrogen peroxide and other derivatives of peroxides, produced physiologically in organisms and occurring in higher concentrations under some pathologic conditions, diffuse into plasma [3]. The level of total peroxide was measured and was expressed as TOS in this study. The percentage of ratio of the TOS to the TAS gave the OSI, an indicator of the degree of oxidative stress. The evaluation of these oxidative stress parameters can indirectly reflect the changes in microcirculation in the organs during surgery [3].

The major finding of the present study was that plasma OSI was significantly higher at the end of surgery compared to before induction of anesthesia in both groups. The plasma TOS and OSI levels increased significantly with time in both groups, whereas the plasma TAS levels decreased with time in both groups. The TOS and OSI change ratio of propofol group were significantly lower than that of thiopental group ( $P=0.004$ and 0.003 , respectively).

Trauma and surgical injury are associated with increased production of ROS, and the use of antioxidant system, in particular when associated with relative tissue ischemia followed by reperfusion, may inhibit ROS production. Oxi- 
dative stress results from an imbalance between radicalgenerating and radical-scavenging systems, which leads to cell membrane impairment [8].

In the present study, oxidative stress was multifactorial in origin; the main impacts were from the surgical trauma of both laparotomy and gastrotomy, and from ischemiareperfusion events due to viseceral organ manipulation and anesthesia. TOS levels of both experimental groups were increased after surgery. In a similar manner, a recent study on surgical patients reported that surgical trauma alone raises TOS [7].

Blood contains many antioxidant molecules that prevent and inhibit harmful effects of ROS. The effects of measurement of total antioxidant status reflect the antioxidative status of plasma. The cooperation of these various antioxidants in plasma provides protection of the organs against oxidative stress. In the present study, we observed a decrease in TAS from induction of anesthesia to the end of surgery in both groups. These results are evidence for change of antioxidant defense systems under surgical condition. However, there was no statistically significant change between groups. The plasma TOS and OSI levels increased significantly with time in both groups, whereas the plasma TAS levels decreased with time in both groups. The TOS and OSI change ratio of propofol group were significantly lower than that of thiopental group.

The antioxidant effects of propofol have been attributed to its chemical similarity with other known antioxidants such as butylhydroxytoluene and $\alpha$-tocopheral [1]. These substances bind to cell membrane phospholipids and capture free radicals, inhibiting the oxidative stress [5]. Numerous studies have clearly demonstrated the antioxidant effects of propofol in last decades [6,9-11]. Similar to previous studies, the TOS and OSI change ratio in propofol groups were significantly lower than those in thiopental groups in this study.

In conclusion, our results show that propofol has antioxidant effects in dogs. The effects may be beneficial for patients in whom free radicals play an important role, such as those with ischemic processes. Further studies need to be conducted to demonstrate the exact mechanism of oxidative stress due to anesthesia and surgery in dogs.
ACKNOWLEDGMENTS. This work was supported by the National Research Foundation of Korea Grant funded by the Korea Government (Ministry of Education, Science and Technology). [NRF-2010-355-E00058]

\section{REFERENCES}

1. Aarts, L., van der Hee, R., Dekker, I., de Jong, J., Langemeijer, H. and Bast, A. 1995. The widely used anesthetic agent propofol can replace alpha-tocopherol as an antioxidant. FEBS Lett. 357: 83-85. [Medline] [CrossRef]

2. Chen, J. and Mehta, J. L. 2004. Role of oxidative stress in coronary heart disease. Indian Heart J. 56: 163-173. [Medline]

3. Erel, O. 2004. A novel automated direct measurement method for total antioxidant capacity using a new generation, more stable ABTS radical cation. Clin. Biochem. 37: 277-285. [Medline] [CrossRef]

4. Griendling, K. K. and FitzGerald, G. A. 2003. Oxidative stress and cardiovascular injury: Part II: animal and human studies. Circulation 108: 2034-2040. [Medline] [CrossRef]

5. Halliwell, B. 1991. Reactive oxygen species in living systems: source, biochemistry, and role in human disease. Am. J. Med. 91: 14S-22S. [Medline] [CrossRef]

6. Kobayashi, K., Yoshino, F., Takahashi, S. S., Todoki, K., Maehata, Y., Komatsu, T., Yoshida, K. and Lee, M. C. 2008. Direct assessments of the antioxidant effects of propofol medium chain triglyceride/long chain triglyceride on the brain of stroke-prone spontaneously hypertensive rats using electron spin resonance spectroscopy. Anesthesiology 109: 426-435. [Medline] [CrossRef]

7. Koksal, H. and Kurban, S. 2010. Total oxidant status, total antioxidant status, and paraoxonase and arylesterase activities during laparoscopic cholecystectomy. Clinics 65: 285-290. [Medline] [CrossRef]

8. Mishra, V. 2007. Oxidative stress and role of antioxidant supplementation in critical illness. Clin. Lab. 53: 199-209. [Medline]

9. Tesauro, M., Thompson, W. C. and Moss, J. 2006. Effect of staurosporine-induced apoptosis on endothelial nitric oxide synthase in transfected COS-7 cells and primary endothelial cells. Cell Death Differ. 13: 597-606. [Medline] [CrossRef]

10. Wang, B., Luo, T., Chen, D. and Ansley, D. M. 2007. Propofol reduces apoptosis and up-regulates endothelial nitric oxide synthase protein expression in hydrogen peroxide-stimulated human umbilical vein endothelial cells. Anesth. Analg. 105: 1027-1033. [Medline] [CrossRef]

11. Xu, J. J. and Wang, Y. L. 2008. Propofol attenuation of hydrogen peroxide-mediated oxidative stress and apoptosis in cultured cardiomyocytes involves haeme oxygenase-1. Eur. J. Anaesthesiol. 25: 395-402. [Medline] [CrossRef] 